\title{
A Dynamic Approach for Rate Adaptation in Mobile Adhoc Networks
}

\author{
${ }^{1}$ Suganya Subramaniam and ${ }^{2}$ Palaniammal Senniappan \\ ${ }^{1}$ Department of Computer Applications, TamilNadu College of Engineering, Coimbatore, India \\ ${ }^{2}$ Department of Science and Humanities, Sri Krishna College of Technology, Coimbatore, India
}

Received 2012-08-01, Revised 2012-08-16; Accepted 2013-06-08

\begin{abstract}
A Mobile Ad hoc Network (MANET) is a collection of mobile nodes with no fixed infrastructure. The absence of central authorization facility in dynamic and distributed environment affects the optimal utilization of resources like, throughput, power and bandwidth. Rate adaptation is the key technique to optimize the resource throughput. Some recently proposed rate adaptations use Request to Send/Clear to Send (RTS/CTS) to suppress the collision effect by differentiating collisions from channel errors. This study presents a methodology to detect the misbehavior of nodes in MANET and proposed the new dynamic algorithm for rate adaptation which in turn can improve the throughput. The proposed approach is implemented in the distributed stipulating architecture with core and access routers. This method does not require additional probing overhead incurred by RTS/CTS exchanges and may be practically deployed without change in firmware. The collision and channel error occurrence will be detected by core router and intimated to the access router to choose alternate route and retain the current rate for transmission. The extensive simulation results demonstrate the effectiveness of proposed method by comparing with existing approaches.
\end{abstract}

Keywords: Channel Error, Collision Control Mechanism, Distributed Architecture, Resource Optimization

\section{INTRODUCTION}

The key idea of multi rate adaptation schemes is to find the data rate that can maximize the throughput and its associated packet delivery ratio. Seok et al. (2003) stated that the multi rate adaptation algorithms in a mobile adhoc network is also to allocate the maximum possible transmission rates to nodes. In this approach, Multiple Access Interference (MAI) is minimized while receiving nodes which is able to decode the transmitted packets given in the current channel state. But, developing an efficient multi rate adaptation scheme in multi hop mobile ad hoc networks is challenging. Because the accurate assessment of the channel misbehaviors like collision, channel error in multi hop mobile ad hoc networks is more difficult than a fixed infrastructure mode network. Whereas many solutions exist for the rate adaptation problem, the design of an efficient solution applicable to many scenarios has proven to be intangible. To some extent, this is due to the complex nature of a wireless channel and the mobility of nodes. Most of the suggested approaches can not distinguish the problem for packet loss while upgrading the transmission rate. Those algorithms immediately decrease the transmission rate to overcome the problem. But when a packet is lost due to channelerrors, an efficient multi rate adaptation algorithm at the sender of the frame would reduce its transmission rate to reduce the channel-errors. However, if the loss is due to a collision, the reduction in the transmission rate would increase the delivery time of the packets and resulting in an increased probability of collisions (Heusse et al., 2005). The solution to rate adaptation presented in this study carefully considers the reasons for packet loss in a multi hop mobile ad hoc network and revolutionize the data rate accordingly. The Coimbatore, India 
proposed algorithm is implemented in the stipulating distributed architecture in that the core router would identify the reason for packet loss and intimate to access routers to handle that situation. The algorithm was implemented in access routers to upgrade the data rate as per the instruction given by core router. The methodology proposed for identifying collision and channel errors where implemented in core routers. As expected, our experiments show that the existing multi rate adaptation algorithms are troubled by collisions and channel errors in rate selection.

\section{MATERIALS AND METHODS}

\subsection{Historical Background}

Several rate adaptation algorithms have been developed over the past decades. Most of the algorithms adjust their transmission rate with the help of the channel feedback inferred from MAC layer retransmission/loss counts. Cardenas et al. (2004) stated that the basic idea of rate selection is to estimate the channel condition and adaptively select the best rate out of multiple available transmission rates. Among the existing rate adaptation algorithms, the earliest and the most widely used one is Auto-Rate Fallback (ARF) proposed by Kamerman and Monteban (1997). This algorithm follows trial and error approach to select the optimum modulation scheme. The data transmission is first tried with a high bit rate modulation scheme and reduced in cases of transmission errors. But, such schemes may not be suitable when the channel is rapidly fluctuating because performance and efficiency of rate adaptation depend on the channel behavior. Moreover this algorithm cannot be distinguished whether that losses are due to channel errors or collision.

Lacage et al. (2004), proposed the Adaptive ARF (AARF) algorithm which performs as ARF with the difference, that the number of consecutive successful transmission attempts is incremented exponentially that every time the higher rate transmission fails. AARF performs better than ARF in case of single-user scenarios, but not in multi-user scenarios. Wong et al. (2006), is implemented the Robust Rate Adaptation Algorithm in two different phases that the rate selector, which selects the transmission rate, based on the computation of the packet loss and the Adaptive RTS, which turns on and off the RTS/CTS mechanism based on the results of the last transmission. But the parameter of the rate selector depends on the used rate and the authors proposed default values for them. ReceiverBased Auto Rate (RBAR) is a receiver-based multi rate algorithm proposed by Holland et al. (2001). It assumes to use the RTS/CTS handshaking. When receiving an RTS frame, the receiver estimates the channel quality based on the Signal to Interference and Noise Ratio (SINR) of the received RTS frame and then determines the best data rate that the transmitter must use. The estimated optimal rate is then sent back to the sender by piggybacking in the CTS packet. This approach is better than ARF, but involves overhead to handling RTS/CTS frames. The Collision-Aware Rate Adaptation (CARA) scheme proposed by Kim et al. (2006), which makes use of the RTS/CTS mechanism for differentiating the packet loss. CARA implements an adaptive RTS/CTS probing scheme which reduces the overall RTS/CTS usage. They show that their algorithm well performed than ARF in a multi-user scenario, but it is not compared with other mechanisms that are known to perform better, like RBAR.

Qiao et al. (2003) and MacKenzie and Wicker (2003) stated about the energy-efficient rate adaptation that dynamically selects a suitable modulation schemes based on the consideration of channel state and network traffic. The algorithm proposed by Serrano et al. (2010) considers joint energy minimization and throughput maximization in the circumstance where the users are able to decide their power and rate combination depending on their interest. This approach is focus on the individual users endeavor for their utility maximization at the expense of others. Most of the rate adaptation algorithms centered on the time-varying characteristics of wireless channels, pay no attention to the impact of collisions. Thus, the algorithm may reduce the transmit rate in the case of frame collisions resulting in unnecessary rate down shift even when channel noise is low.

This can significantly decrease throughput when transmission failures are caused by collisions as specified by Reis et al. (2006). Hence the above mentioned papers propose various algorithms to identify the optimal rate for transmission. This study proposes a new algorithm which differentiates the channel error and Collision occurrence in the transmission to increase or decrease the transmission rate which in turn can improve the throughput. This method does not required additional probing overhead incurred by RTS/CTS exchanges and the results compared with ARF and RBAR approaches.

\subsection{Architecture Used for Proposed Algorithm}

The algorithm proposed in this study is differentiates the misbehavior of transmission channel and upgrade the 
transmission rate accordingly. The proposed approach was implemented in the dynamic distributed architecture. Assume that the architecture composed by core and access routers, as shown in Fig. 1. Traffic monitors are installed on access and core routers to perform online measurements on the incoming traffic flows. Collision detection algorithm is implemented in the core routers to detect the collision occurrence in the channel with the help of receiver node. Core routers send messages with access routers to report about the collision or any other channel errors. Our proposed dynamic rate adaptation algorithm is deployed in all access routers and sender node.

Each access router collects the measurements performed by traffic monitors and information reported by core routers regarding the channel misbehavior. The proposed algorithm works based on this information to upgrade the data rate of the sender host or decides on alternate route and exchange update messages with all other access routers to report the current incoming traffic statistics.

\subsection{Differentiating Collisions and Channel Errors}

The selection of optimum transmission rate depends on the channel quality. If the sender chooses a higher rate than the current channel can support, the packet is at risk to noise. If the sender chooses a rate that is lower than the channel can support, the channel utilization is low. Consecutively it reduces the throughput. Most of the rate adaptation algorithms achieve this by comparing the number of successful and unsuccessful transmissions. The successful transmission is identified with the ACK from the receiver. If an ACK is not received, the packet is considered to be lost due to channel errors, not estimated the exact grounds for packet loss. Since the up gradation in transmission rate is depends on the cause of packet loss, the first stage of the proposed approach is to identify the exact cause.

\subsection{Packet Loss Due to Channel Errors}

Qiu et al. (2007) has shown that, in a wireless channel, the packet bursts due to interference or noise and there are some parts of the packet that can be decoded correctly as shown in Fig. 2.

Depending on the statistical assumptions about channel errors, each separate bit is subject to error with a certain probability. Such a pattern of irregular errors in a packet can be used as an indication that the packet was lost due to channel errors.

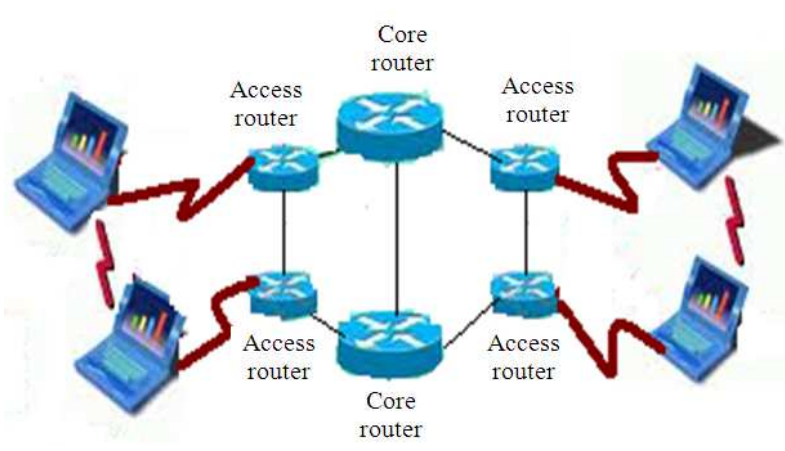

Fig. 1. Dynamic distributed architecture

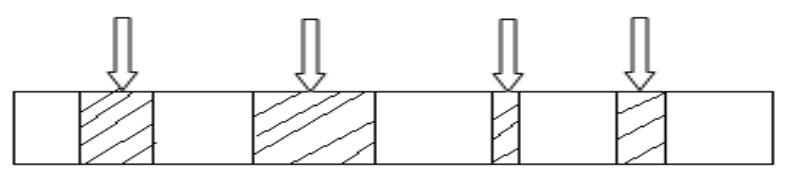

Fig. 2. Packet with lossy information

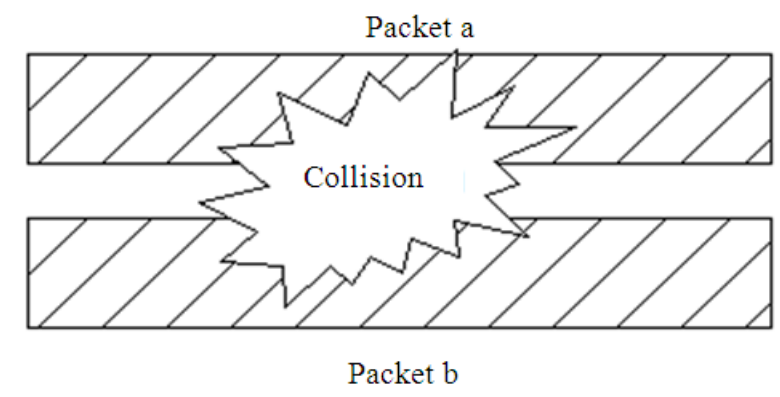

Fig. 3. Collision occurrence

\subsection{Packet Loss Due to Collisions}

A collision is the situation that occurs when two or more devices attempt to send a signal along the same transmission channel at the same time. In that case, all packets of involved transmitters will be corrupted completely, including their headers (Fig. 3). The chances for collision to happen are proportional to the number of nodes in the area.

\subsection{Congestion Control Mechanism}

In this study a new congestion control mechanism is implemented by accessing the information provided by $\mathrm{Fu}$ et al. (2003) and Whitehouse et al. (2005), which takes router-assisted approach to dynamically adjust the size of congestion window according the 
router information that reduces collisions and achieves the maximum throughput. In the proposed architecture the core router first measures the lingering bandwidth and RoundTripTime (RTT) of each transmission. To perform these functions, the router has to maintain two timers: one timer for recording the packet interarrival interval and the other for measuring the RTT. The sender makes use of this information to adjust its congestion window.

The available residual bandwidth in each router depends on many factors such as the length of the queue, queuing time, buffer size and length of the spare queue. This study implements the method suggested by Suganya and Palaniammal (2009) to estimate the available lingering bandwidth.

Let $B_{w}$ be the $i$-th measured bandwidth. Then, the bandwidth can be continuously updated by:

$$
\mathrm{B}_{\mathrm{w}}=\propto \mathrm{B}_{\mathrm{w}}+(1-\propto) \mathrm{B}_{\mathrm{w}_{\mathrm{i}}}
$$

where, $\alpha$ is an exponential filter co-efficient, to reduce the influence of cross traffic, a low pass filter can also be adopted.

The measurement of the RTT is based on the data packet arrival time. A variable rcv.rtt is used to record the RTT.

The algorithm can be described as follows:

- When an ACK is sent, if rev.rtt is zero, let rev.rtt equal 1 and record the corresponding sequence (rtseq) of data packet (equals to the sum of the ACK sequence and the current congestion window). Otherwise, just send the ACK

- If a data packet with a larger sequence number than "rtseq" is arrived in order and the new measured packet inter-arrival interval is larger than two times of pre-estimated packet inter-arrival interval, set the new measured RTT to the value of rev.rtt and let rev.rtt be zero

- On each clock cycle, if rcv.rtt is not zero, rcv.rtt is added by 1 . Then, the retransmission timer is set based on the measured RTT

The receiver acquires the bandwidth measure and RTT to calculate the congestion window value rwnd as:

$$
\text { rwnd }=\mathrm{B}_{\mathrm{w}} * \mathrm{RTT}
$$

Then, it compares the rwnd with the available receiver buffer and deposits the lesser value in the advertised window field of an ACK going back to the sender:

$$
\operatorname{adv} \_w n d=\min \left(r \_a b u f, \text { rwnd }\right)
$$

In other words, the receiver advertised window not only has the original flow control function, but also takes on the congestion control function.

When the receiver detects a timeout, it will send an ACK to inform the sender that the network is congested. In this ACK, the receiver advertised window is set to one packet, meaning that the sender should retransmit the dropped packet.

If the receiver must have detected this timeout earlier than the sender, it will help the sender to reduce the waiting time and confirm the packet loss.

The receiver and core router helps the sender to increase the congestion window according to the instantaneous available bandwidth. The sender only needs to maintain the AIMD mechanism in the congestion avoidance stage and the slow start stage can be eliminated. Once the sender received the information from core router or receiver, it will decrease the congestion window to one in consideration that the network is in congestion and it will have some time to recover.

If congestion is mitigated after one RTT, the sender will recover by adjusting the congestion window in the next transmission by using the receiver advertised window. During fast retransmission, the sender sets the congestion window size to the lesser value of the receiver advertise window size and the current size. Since, if the congestion window is less than the receiver advertised window, the network may only be in a mild congestion.

\subsection{Proposed Dynamic Data Rate Adaptation Algorithm (DRAA)}

With the exemption of few algorithms, all approaches have a common characteristic that reduce the data rate if any misbehavior in the channel. But in the proposed approach, core router identifies exactly the cause of the packet drop and suggests the sender to increase or decrease the data rate. 
The stepwise procedure of proposed approach for dynamic updating in data rate is as follows:

- At the source node, establish the route and transmit the packet with data rate recommended at the current state for transmission

- Repeat the following steps in periodical interval $t$

- Collect the information from core router and access router in the regard of channel position

- If no misbehavior is found in the channel the sender increases the data rate and continues the transmission in the same channel

- Else based on the information from access or core router identify the exact cause for misbehavior. If channel error occurs then choose the alternate path

- If the core router intimates about the occurrence of collision in the channel with the assist of the algorithm already described which suggests for increasing the congestion window as well as increase the data rate, since the data rate increases then it will reduce the congestion rate as proposed by Rayanchu et al. (2008)

\section{RESULTS}

In order to evaluate the performance of the proposed algorithm, run simulations using the NS-2 network simulator, version 2.33. During a simulation, the initial data rate depends on the channel position recommended by receiver. The information from the sender is sent with equal size of packets ( 512 bytes). The algorithm for congestion control is implemented in the core router to identify the congestion. The core router receives the message in this regard and intimate to the nodes involved in the transmission. Core router also monitors the channel misbehaviors and intimate to sender. The proposed algorithm DRAA is implemented in the sender node and access router to update the data rate or choose alternate path for attains high throughput. Fig. 4 depicts the simulation scenario for implementing proposed algorithm with 66 numbers of nodes and Table $\mathbf{1}$ defines the parameters used in this study. The Fig. 5 shows that the source node 0 identifies alternate route for the destination node 8 when the link 1-3 gets faded.

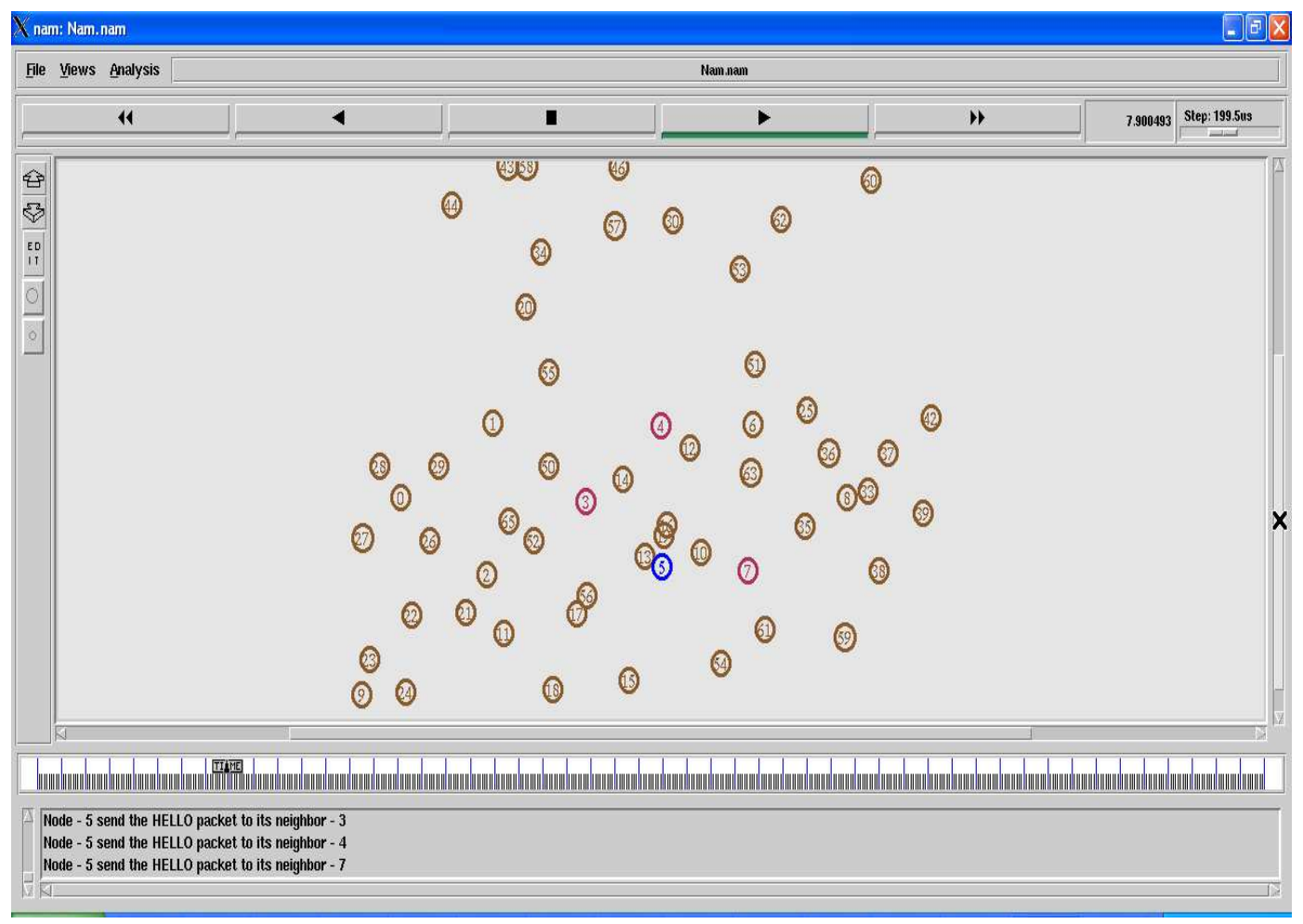

Fig. 4. Simulation scenario 


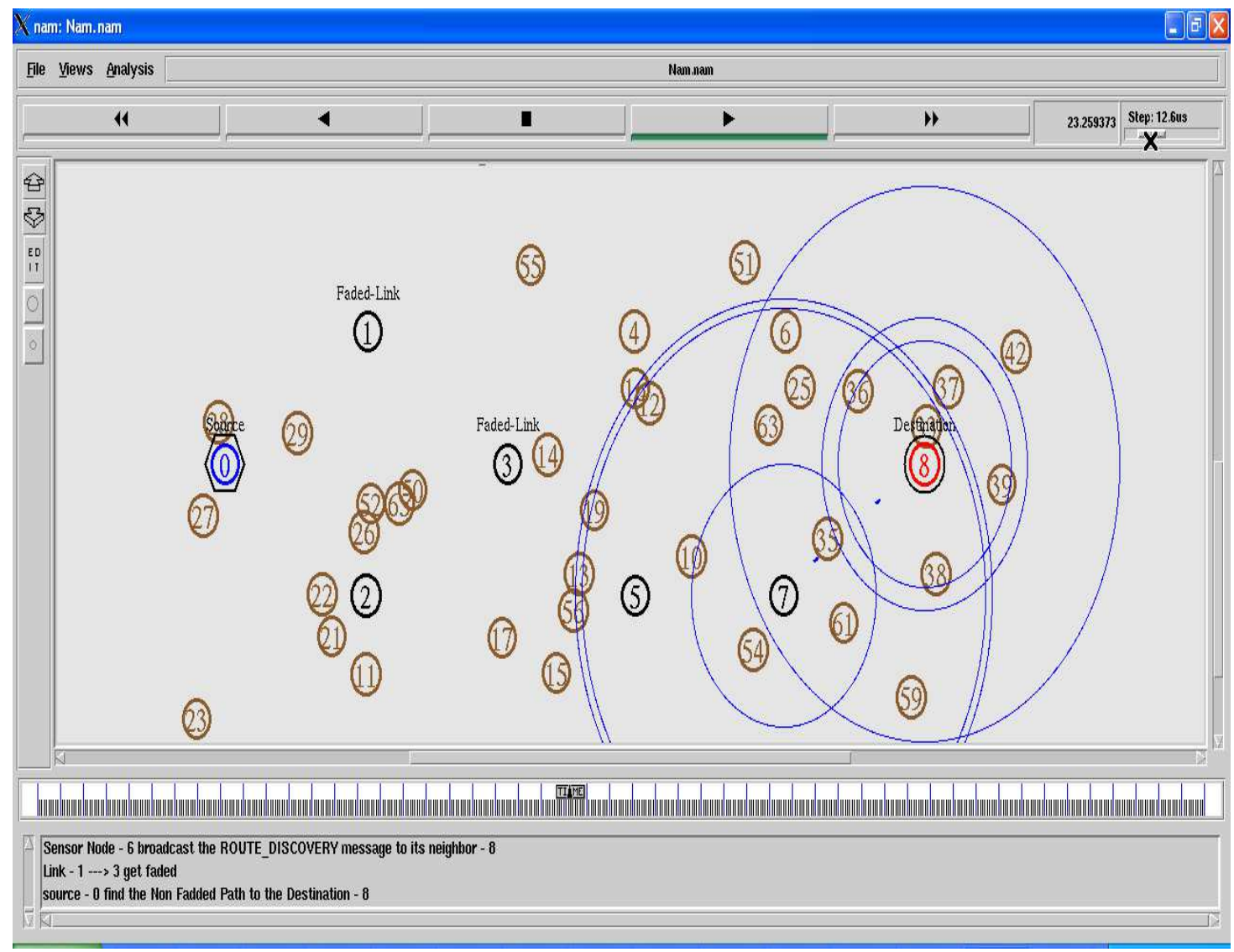

Fig. 5. Broadcasting Route-Discovery message to find new path

Table 1. Parameters for simulation

\begin{tabular}{ll}
\hline Parameter & Value \\
\hline Data Packet size & 512 bytes \\
Communication range & $250 \mathrm{~m}$ \\
Carrier sensing range & $550 \mathrm{~m}$ \\
Queue Length & 500 \\
Frequency & $9 \mathrm{MHz}$ \\
Routing protocol & AOMDV \\
Grid size & $1000 \times 1000 \mathrm{~m}$ \\
\hline
\end{tabular}

\section{DISCUSSION}

\subsection{Performance Comparisions of DRAA with Existing Approaches}

Simulation results illustrate in Fig. 6a and $\mathbf{b}$ that DRAA algorithm increases both packet delivery ratio and throughput with respect to ARF and RBAR. RBAR performs always worse than ARF because of the additional probing overhead incurred by RTS/CTS exchanges. As expected, both ARF and RBAR not perform well when the time goes on. The reason, as explained before, is that these algorithms not distinguish the exact cause for packet loss. So, the algorithms always decrease the data rate when the channel misbehaves.

The Fig. 7a and b signifies the End to End Delay and Packet Drop of proposed approach DRAA and the other two approaches ARF, RBAR. End to End Delay refers the average time taken by a data packet to arrive in the destination. It also includes the delay caused by route discovery process and the queue in data packet transmission. Initially all three approaches are in the same position, but the proposed approach have less delay time as well as less packet drop when time goes on.

The Fig. 8 illustrates the comparison in consumption of the resource power in the proposed approach and the other two schemes. It depicts that the DRAA consumes lesser than the other approaches. 
Suganya Subramaniam and Palaniammal Senniappan / American Journal of Applied Sciences 10 (6): 596-605, 2013

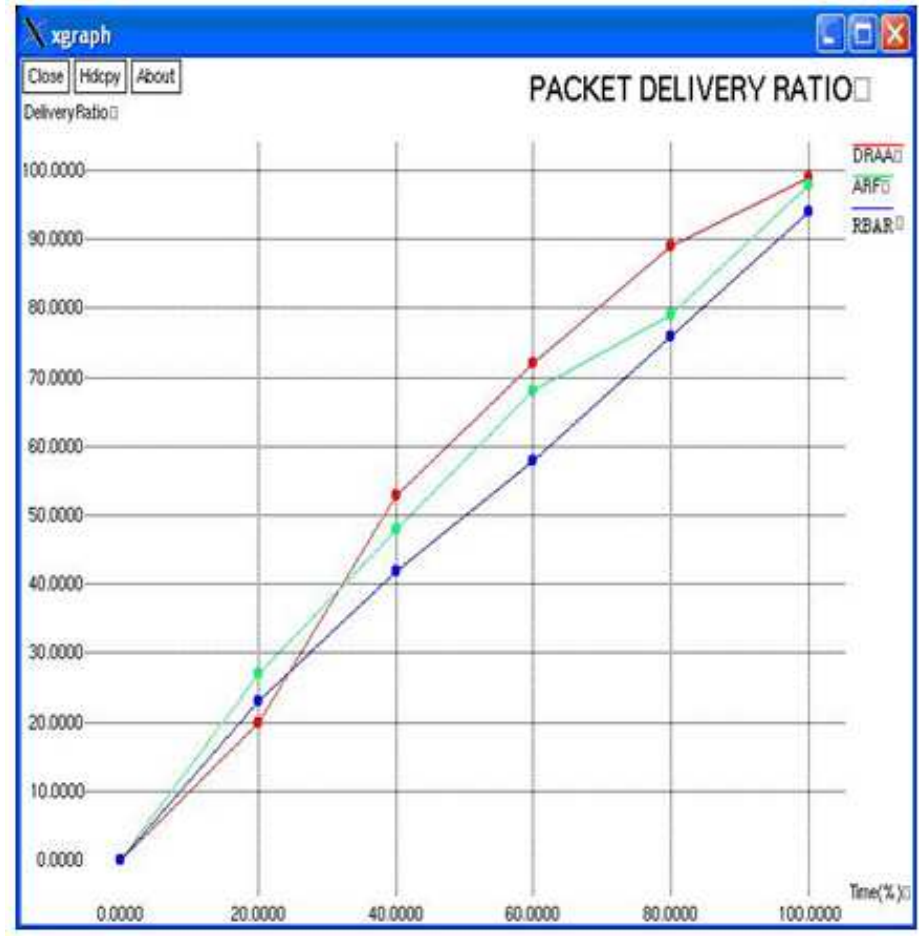

(a)

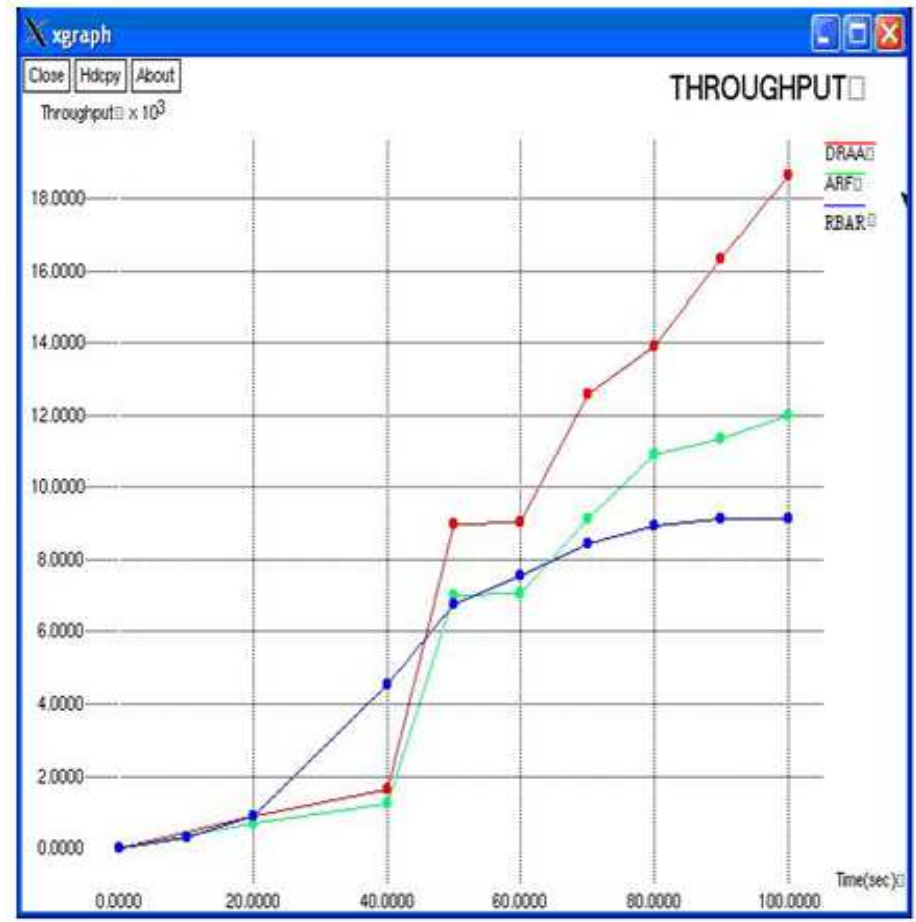

(b)

Fig. 6. (a) Packet Delivery Ratio (b) Throughput 
Suganya Subramaniam and Palaniammal Senniappan / American Journal of Applied Sciences 10 (6): 596-605, 2013

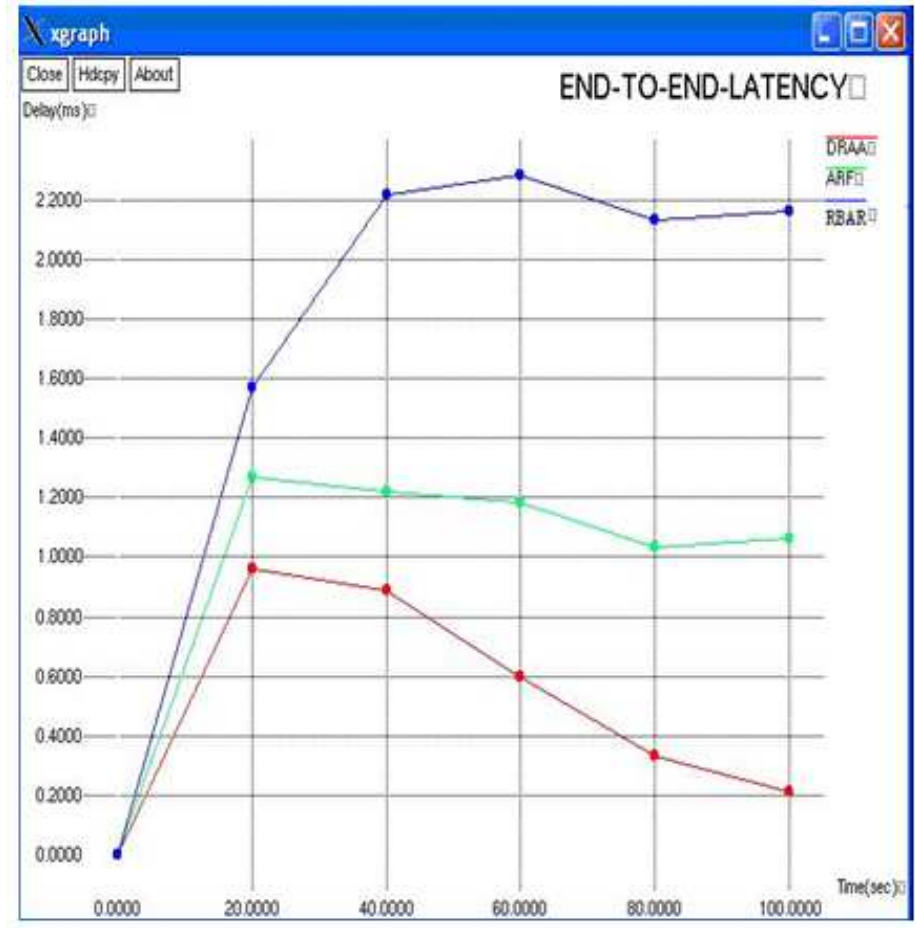

(a)

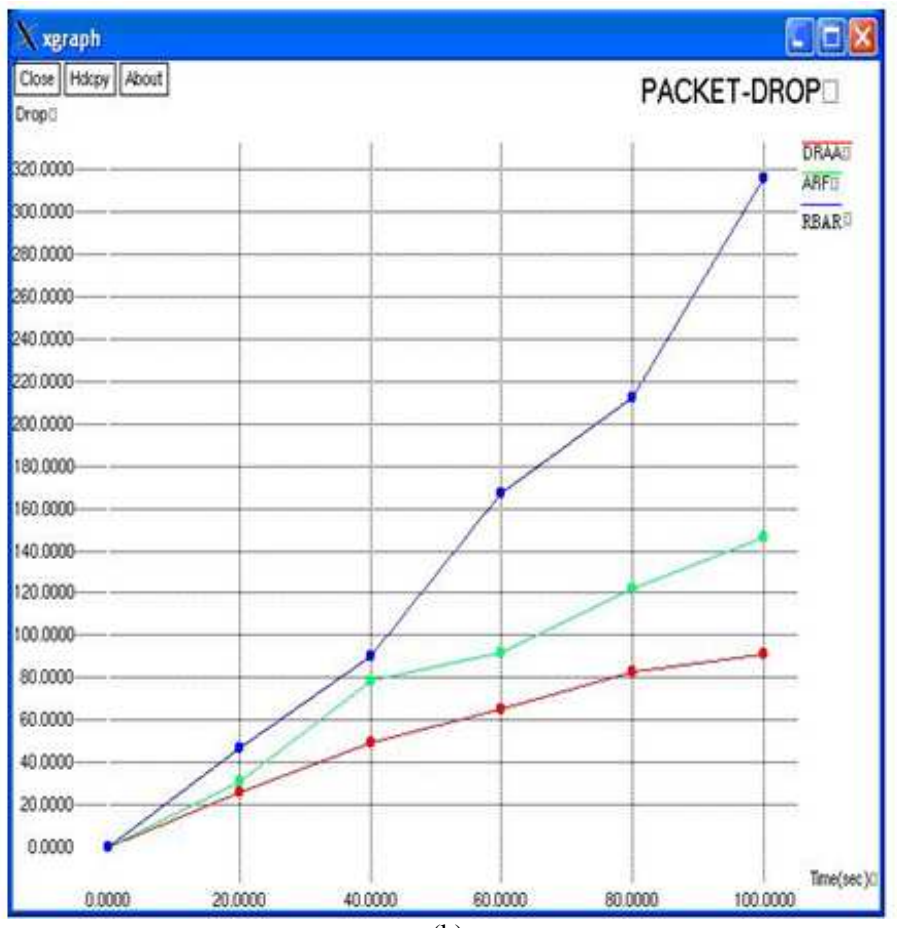

(b)

Fig. 7. (a) End-to-End Delay (b) Packet Drop 


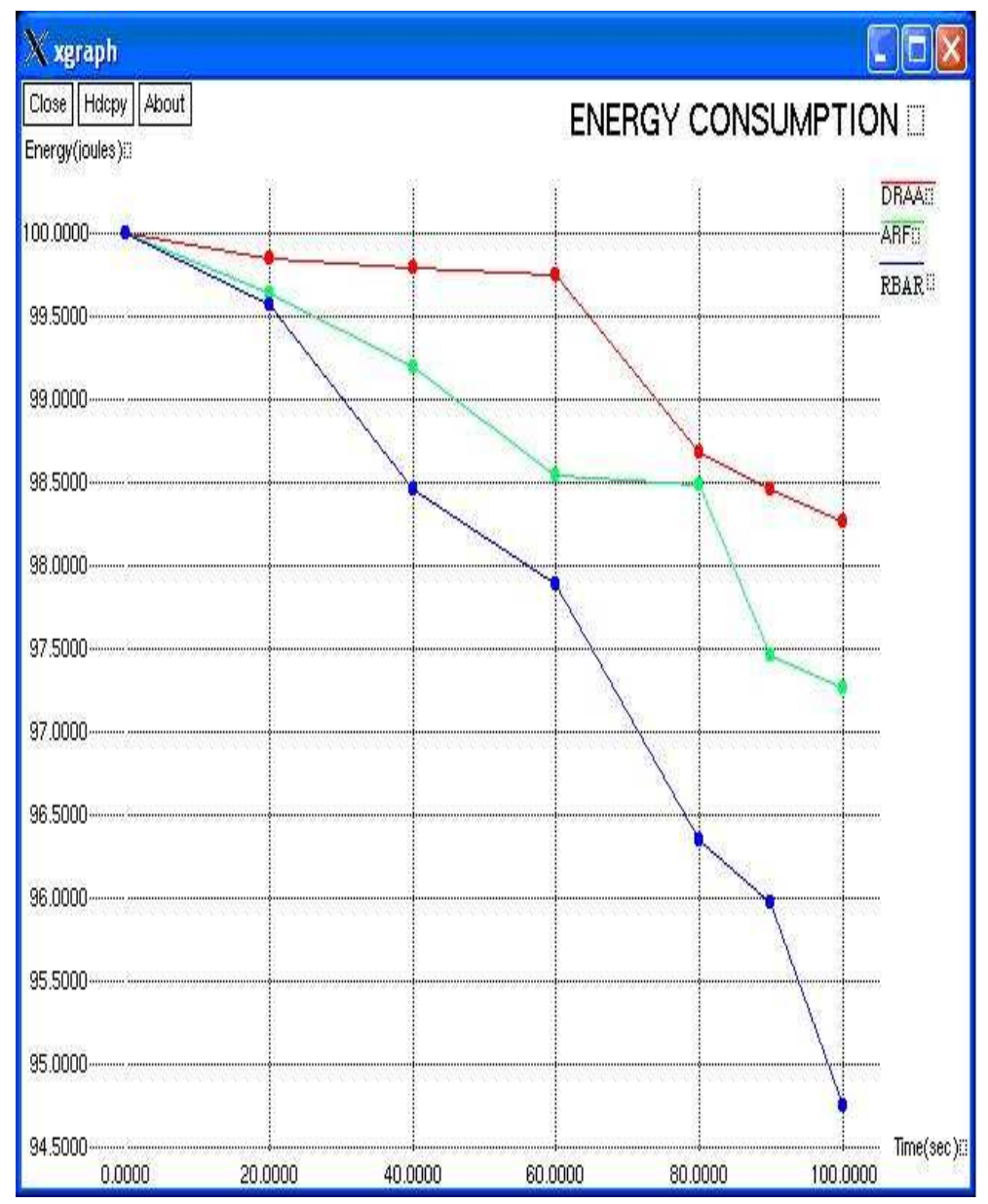

Fig. 8. Power consumption

\section{CONCLUSION}

This study discusses the methodology to distinguish the misbehavior of channel in multi hop mobile adhoc networks and presents a new dynamic rate adaptation algorithm depending on the channel behavior. A key idea of the proposed approach is to increase or decrease the data rate depends on channel position. Therefore the sender node is conversant with the exact cause for packet loss to decide about the up gradation of data rates. This results in maximize the key resource throughput as well as packet delivery ratio. Simulation results also shows the less delay time and packet drops in the proposed approach. Further, proposed model may consider different properties of network nodes (e.g., energy consumption). As a conclusion that the proposed approach performs so well since it can be implemented with minimal overhead.

\section{REFERENCES}

Cardenas, A.A., S. Radosavac and J.S. Baras, 2004. Detection and prevention of MAC layer misbehavior in ad hoc networks. Proceedings of the 2nd ACM Workshop on Security of Ad Hoc and sensor Networks, Oct. 25-29, ACM Press, Washington, DC, USA., pp: 17-22. DOI: 10.1145/1029102.1029107

Fu, Z., P. Zerfos, H. Luo, S. Lu and L. Zhang et al., 2003. The impact of multihop wireless channel on TCP throughput and loss. Proceedings of the IEEE Societies 22nd Annual Joint Conference of the IEEE Computer and Communications, Mar. 30-Apr. 03, IEEE Xplore Press, pp: 1744-1753. DOI: 10.1109/INFCOM.2003.1209197 
Heusse, M., F. Rousseau, R. Guillier and A. Duda, 2005. Idle sense: An optimal access method for high throughput and fairness in rate diverse wireless LANs. Proceedings of the Conference on Applications, Technologies, Architectures and Protocols for Computer Communications, Aug. 2226, ACM Press, Philadelphia, PA, USA., pp: 121132. DOI: $10.1145 / 1080091.1080107$

Holland, G., N. Vaidya and P. Bahl, 2001. A rateadaptive MAC protocol for multi-Hop wireless networks. Proceedings of the 7th Annual International Conference on Mobile Computing and Networking, Jul. 16-21, ACM Press, Rome, Italy, pp: 236-251. DOI: $10.1145 / 381677.381700$

Kamerman, A. and L. Monteban, 1997. WaveLAN ${ }^{\circledR}$-II: A high-performance wireless LAN for the unlicensed band. Bell Labs Technical J., 2: 118-133. DOI: $10.1002 /$ bltj.2069

Kim, J., S. Kim, S. Choi and D. Qiao, 2006. CARA: Collision-aware rate adaptation for IEEE 802.11 WLANs. Proceedings of the 25th IEEE International Conference on Computer Communications, (ICCC' 06), IEEE Xplore Press, Barcelona, Spain, pp: 1-11. DOI: $10.1109 /$ INFOCOM.2006.316

Lacage, M., M.H. Manshaei and T. Turletti, 2004. IEEE 802.11 rate adaptation: A practical approach. Proceedings of the 7th ACM International Symposium on Modeling, Analysis and Simulation of Wireless and Mobile Systems, Oct. 4-6, ACM Press, Venice, Italy, pp: 126-134. DOI: 10.1145/1023663.1023687

MacKenzie, A.B. and S.B. Wicker, 2003. Stability of multipacket slotted Aloha with selfish users and perfect information. Proceedings of the IEEE Societies 22nd Annual Joint Conference of the IEEE Computer and Communications, Mar. 30-Apr. 03, IEEE Xplore Press, pp: 1583-1590. DOI: 10.1109/INFCOM.2003.1209181

Qiao, D., S. Choi, A. Jain and K.G. Shin, 2003. MiSer: An optimal low-energy transmission strategy for IEEE $802.11 \mathrm{a} / \mathrm{h}$. Proceedings of the 9 th Annual International Conference on Mobile Computing and Networking, Sept. 14-19, ACM Press, San Diego, CA, USA., pp: 161-175. DOI: 10.1145/938985.939003

Qiu, L., Y. Zhang, F. Wang, M.K. Han and R. Mahajan, 2007. A general model of wireless interference. Proceedings of the 13th Annual ACM International Conference on Mobile Computing and Networking, Sept. 9-14, ACM Press, Montreal, Canada, pp: 171182. DOI: $10.1145 / 1287853.1287874$
Rayanchu, S., A. Mishra, D. Agrawal, S. Saha and S. Banerjee, 2008. Diagnosing wireless packet losses in 802.11: Separating collision from weak signal. Proceedings of the IEEE 27th Conference on Computer Communications, Apr. 13-18, IEEE Xplore Press, Phoenix, AZ., pp: 735-743. DOI: 10.1109/INFOCOM.2008.124

Reis, C., R. Mahajan, M. Rodrig, D. Wetherall and J. Zahorjan, 2006. Measurement-based models of delivery and interference in static wireless networks. Proceedings of the Conference on Applications, Technologies, Architectures and Protocols for Computer Communications, Sept. 11-15, ACM Press, Pisa, Italy, pp: 51-62. DOI: 10.1145/1159913.1159921

Seok, Y., J. Park and Y. Choi, 2003. Multi-rate aware routing protocol for mobile ad hoc networks. Proceedings of the 57th IEEE Semiannual Vehicular Technology Conference, Apr. 22-25, IEEE Xplore Press, pp: 1749-1752. DOI: 10.1109/VETECS.2003.1207123

Serrano, P., M. Hollick and A. Banchs, 2010. On the trade-off between throughput maximization and energy consumption minimization in IEEE 802.11 WLANs. J. Commun. Netw., 12: 150-157.

Suganya, S. and S. Palaniammal, 2009. Lingering bandwidth estimation to optimize the resource utilization for mobile adhoc networks. Int. J. Netw. Commun. Eng. DOI: NCE092009005

Whitehouse, K., A. Woo, F. Jiang, J. Polastre and D. Culler, 2005. Exploiting the capture effect for collision detection and recovery. Proceedings of the 2nd IEEE Workshop on Embedded Networked Sensors, May 30-31, IEEE Xplore Press, pp: 45-52. DOI: 10.1109/EMNETS.2005.1469098

Wong, S.H.Y., H. Yang, S. Lu and V. Bharghavan, 2006. Robust rate adaptation for 802.11 wireless networks. Proceedings of the 12th Annual International Conference on Mobile Computing and Networking, Sept. 24-29, ACM Press, Los Angeles, CA, USA., pp: 146-157. DOI: 10.1145/1161089.1161107 